\title{
Ghost spins and quantum critical behavior in a spin chain with local bond deformation
}

\author{
Jianhui Dai \\ Abdus Salam International Center for Theoretical Physics, Trieste 34100, Italy \\ and Zhejiang Institute of Modern Physics, Zhejiang University, Hangzhou 310027, China \\ Yupeng Wang \\ Institut für Physik, Universität Augsburg, D-86135 Augsburg, Germany \\ and Laboratory of Ultra-Low Temperature Physics \& Center for Condensed Matter Physics, Chinese Academy of Sciences, \\ Beijing 100080, China

\section{U. Eckern} \\ Institut für Physik, Universität Augsburg, D-86135 Augsburg, Germany
}

(Received 7 August 1998; revised manuscript received 25 November 1998)

\begin{abstract}
We study the impurity-induced critical behavior in an integrable $S U(2)$-invariant model consisting of an open spin chain of arbitrary spin $S$ (Takhatajian-Babujian model) interacting with an impurity of spin $\vec{S}^{\prime}$ located at one of the boundaries. For $S=1 / 2$ or $S^{\prime}=1 / 2$, the impurity interaction takes a very simple form $J \vec{S}_{1} \cdot \vec{S}^{\prime}$ that describes the deformed boundary bond between the impurity $\vec{S}^{\prime}$ and the first bulk spin $\vec{S}_{1}$ with an arbitrary coupling strength $J$. For a weak coupling $0<J<J_{0} /\left[\left(S+S^{\prime}\right)^{2}-1 / 4\right]$, the impurity is completely compensated, undercompensated, and overcompensated for $S^{\prime}=S, S^{\prime}>S$, and $S^{\prime}<S$ as in the usual Kondo problem. While for strong coupling $J \geqslant J_{0} /\left[\left(S+S^{\prime}\right)^{2}-1 / 4\right]$, the impurity spin is split into two ghost spins. Their cooperative effect leads to a variety of new critical behaviors with different values of $\left|S^{\prime}-S\right|$. [S0163-1829(99)03530-4]
\end{abstract}

\section{INTRODUCTION}

Quantum fluctuations induced by an impurity coupled with the one-dimensional (1D) Tomonaga-Luttinger liquid (TLL) play essential roles in understanding the low temperature behavior of quasi-1D systems, such as quantum wires, ${ }^{1}$ fractional quantum Hall effect, ${ }^{2}$ carbon nanotubes, ${ }^{3}$ or quasi-1D organic conductors. ${ }^{4}$ The problem of an impurity spin $\vec{S}^{\prime}$ coupled with both of its neighboring sites in a quantum chain was studied by a class of integrable SU(2)invariant models. ${ }^{5,6}$ For the Heisenberg chain with ferromagnetic coupling, the impurity is locked into the critical behavior of the lattice, i.e., at low temperatures the specific heat is proportional to $T^{1 / 2}$ and the susceptibility diverges as $T^{-2}$ with logarithmic corrections. ${ }^{7}$ For a chain with antiferromagnetic coupling, the impurity spin is compensated by bulk spins with three different situations similar to those of the multichannel Kondo problem: ${ }^{8}$ for $S^{\prime}=S$, it is the complete compensation and the impurity just corresponds to one more site in the chain; for $S^{\prime}>S$, the partial compensation with Schottky anomaly when an external magnetic field $H$ is applied on; and for $S^{\prime}<S$, the overcompensation, which gives rise to quantum critical behavior. ${ }^{8}$

The effects of an impurity embedded in 1D TLL have been recently extensively discussed. By renormalizationgroup (RG) techniques, bosonization methods, and boundary conformal field theories, many interesting results have been obtained, showing unusual properties of TLL in the presence of a local potential barrier or a magnetic impurity. ${ }^{9-12}$ Generally speaking, these new findings indicate that the quantum impurity models renormalize to critical points corresponding to conformally invariant boundary conditions. ${ }^{13-16}$ The impurity-bulk coupling strength $J$ flows either to infinity when the impurity is screened, or to finite as if it is overscreened, no matter what the sign of $J$ is initially. In particular, numerical studies of the finite-size spectrum support the picture that the fixed point corresponds to a chain disconnected at the impurity site for repulsive interaction. ${ }^{17}$ However, the low-temperature impurity behavior described by previous Bethe ansatz integrable models do not correspond to the stable critical points mentioned above. For instance, at the critical point the spin- $S$ impurity coupled to the spin-1/2 antiferromagnetic Heisenberg chain has the effective screened spin $S_{\text {eff }}=S-1 / 2$ rather than $S-1$, despite the fact that it couples with two neighboring $1 / 2$ spins. ${ }^{5}$ In this respect, the critical point described by the integrable impurity models is unstable, owing to the fact that these models have a fixed impurity coupling, a "fine-tuned" impurity interaction term, and no backward scattering. ${ }^{18}$ It is recalled that backward scattering is one of the essences of the quantum impurity problem in 1D TLL. ${ }^{9,11}$ From the point of view of $\mathrm{RG}$, electrons or spin waves moving in one-dimensional space will be largely scattered back by the impurity, while the tunneling effect could be perturbed. ${ }^{11,19}$ In the fixed-point limit, they are completely scattered back after a phase shift by the impurity, as long as the tunneling is plausibly neglected at sufficient low temperature. We remark that in these models only one channel host electron is treated (forward scattering for the transparent impurity, and one halfchain for the boundary impurity). For a general impurity (nonintegrable), one should consider two half-chains interacting symmetrically with the impurity, ${ }^{22}$ which is effectively a two-channel one ${ }^{18,23}$ at low-energy scales, as long as 
the tunneling across the impurity is plausibly neglected (RG fixed point). Generally, tunneling through the impurity may exist. It causes hybridization, splitting, and anisotropy of the two channels. However, the tunneling matrix is negligibly small comparing to the Kondo coupling ${ }^{11}$ or the impurity potential $^{9}$ at low-energy scales from the RG point of view. Therefore, these effects are not very harmful to the twochannel behavior. ${ }^{24}$ The two-channel Kondo behavior is really found in the spin-1/2 Heisenberg chain with a spin-1/2 impurity. ${ }^{25}$

In this paper, we solve a related integrable model, i.e., the Takhatajian-Babujian spin chain coupled with a boundary spin, via algebraic Bethe ansatz. It is argued that for $S=1$, the model corresponds to a bulk impurity in a spin-1/2 Heisenberg chain. The structure of the present paper is the following: In the subsequent section, we construct the model and derive the Bethe ansatz equation. The ground-state properties and the boundary bound states are discussed in Sec. III. In Sec. IV, we discuss the thermodynamics of the open boundary as well as the impurity. It is found that the open boundary behaves as an overscreened spin and the impurity itself, may show different quantum critical behaviors, depending on the coupling constant $J$. Section $\mathrm{V}$ is attributed to the concluding remarks.

\section{THE MODEL AND ITS BETHE ANSATZ}

Let us start with the following Hamiltonian: ${ }^{25}$

$$
H=J_{0} \sum_{n=1}^{N-1}\left[\vec{S}_{n} \cdot \vec{S}_{n+1}+\vec{S}_{-n} \cdot \vec{S}_{-n-1}\right]+J \vec{S}^{\prime} \cdot\left(\vec{S}_{1}+\vec{S}_{-1}\right),
$$

where $J_{0}>0$ (antiferromagnetic coupling); $\vec{S}_{n}$ is the spin-1/2 operator, $\vec{S}^{\prime}$ is the impurity spin operator. By inverting the coordinates of one half-chain, we readily map the model onto a two-channel spin system coupled with a boundary impurity. As discussed in earlier works, ${ }^{6,24}$ a $2 S$-channel Kondo system has the same low-temperature behavior to that of spin- $S$ Takhatajian-Babujian mode ${ }^{26}$ with an impurity spin. ${ }^{6}$ Therefore, the model (1) has similarity to the following Hamiltonian:

$$
H=J_{0} \sum_{n=1}^{N-1}\left[\vec{S}_{n} \cdot \vec{S}_{n+1}-\left(\vec{S}_{n} \cdot \vec{S}_{n+1}\right)^{2}\right]+J \vec{S}^{\prime} \cdot \vec{S}_{1},
$$

with $S=1$. Based on these arguments, we study the lowtemperature behavior induced by an magnetic impurity $\vec{S}^{\prime}$ coupled with an open antiferromagnetic TakhatajianBabujian chain of spin $S$ by use of Bethe ansatz. It is well known that integrable generalization of isotropic $S=1 / 2$ spin chain to arbitrary spin $S$ leads to the Hamiltonian ${ }^{26}$

$$
H_{S}=J_{0} \sum_{j=1}^{N-1} Q_{2 S}\left(\vec{S}_{j} \cdot \vec{S}_{j+1}\right)
$$

where $Q_{2 S}(x)$ is a polynomial of degree $2 S$ of SU(2) invariant quantities $x=\vec{S}_{j} \cdot \vec{S}_{j+1}$,

$$
Q_{2 S}(x)=\sum_{j=1}^{2 S}\left(\sum_{k=1}^{j} \frac{1}{k}\right) \prod_{l \neq j, l=0}^{2 S} \frac{x-x_{l}}{x_{j}-x_{l}},
$$

with $\quad x_{n}=\frac{1}{2} n(n+1)-S(S+1), \quad n=0,1, \ldots, 2 S . \quad$ One recovers $\quad H_{1 / 2}=J_{0} \Sigma_{j=1}^{N-1} \vec{S}_{j} \cdot \vec{S}_{j+1}, \quad H_{1}=J_{0} \Sigma_{j=1}^{N-1}\left[\vec{S}_{j} \cdot \vec{S}_{j+1}\right.$ $\left.-\left(\vec{S}_{j} \cdot \vec{S}_{j+1}\right)^{2}\right]$ as the usual spin-1/2 Heisenberg model and the $S=1$ Takhatajian-Babujian model, respectively (up to an irrelevant constant). The construction of the model is based on the vertex weight operators $R(\lambda)$, represented by matrices acting on the tensor product spaces $V_{1} \otimes V_{2}$ of two spins $\vec{S}_{1}, \vec{S}_{2}$, with a parameter $\lambda$ identified as spectral parameter. Its explicit form is

$$
{ }_{S} R^{12}(\lambda)=-\sum_{l=0}^{2 S} \prod_{k=0}^{l} \frac{\lambda-k}{\lambda+k} P_{l},
$$

where $P_{l}$ is the projector selecting the states with total spin $l$ in the tensor product of the two spins involved, $P_{l}(x)$ $=\prod_{n \neq l, n=0}^{2 S}\left(x-x_{n}\right) /\left(x_{l}-x_{n}\right)$. Owing to the Yang-Baxter equations satisfied by the $R$ matrix, we have the relationship $\left.H_{S} \propto(d / d \lambda) \ln t(\lambda)\right|_{\lambda=0}$, with $t(\lambda)$ being the transfer-matrix defined by

$$
t(\lambda)=\operatorname{tr}_{A} T(\lambda)=\operatorname{tr}_{A}\left\{{ }_{S} R^{A N}(\lambda) \cdots_{S} R^{A 1}(\lambda)\right\} .
$$

Here, the trace is taken in the auxiliary spin space $V_{A}$ $\left(\operatorname{dim} V_{A}=2 S+1\right)$ introduced to help us track the proliferating spin indices. Because $[t(\lambda), t(\mu)]=0, \forall \lambda, \mu, H_{S}$ is integrable under the periodic boundary condition.

Now, we put a magnetic impurity $\vec{S}^{\prime}$ at one end of the chain, by considering the following integrable Hamiltonian

$$
\begin{gathered}
H=H_{S}+H_{\text {imp }}, \\
H_{\text {imp }}=J_{0} \sum_{l=\left|S-S^{\prime}\right|+1}^{S+S^{\prime}}\left(\sum_{k=\left|S-S^{\prime}\right|+1}^{l} \frac{k}{k^{2}-c^{2}}\right) \\
\times \prod_{n \neq l, n=\left|S-S^{\prime}\right|} \frac{y-y_{n}}{y_{l}-y_{n}}
\end{gathered}
$$

where $y=\vec{S}_{1} \cdot \vec{S}^{\prime}, y_{l}=\frac{1}{2}\left[l(l+1)-S(S+1)-S^{\prime}\left(S^{\prime}+1\right)\right] ; c$ is an arbitrary parameter describing the strength of the bulkimpurity interaction. The impurity is assumed to be sited at the left end of the chain, say the site $j=0$, while its neighboring spin is $\vec{S}_{1}$. For $S=1 / 2$, the model is reduced to that considered in Ref. 21. Interestingly, when $S=1 / 2$ or $S^{\prime}$ $=1 / 2$, the interaction term takes the simple form

$$
H_{i m p}=J \vec{S}_{1} \cdot \vec{S}^{\prime},
$$

with coupling constant $J=J_{0} /\left[\left(S^{\prime}+S\right)^{2}-c^{2}\right]$, which can range from negative infinity to positive, and meet all the physical situations. So at least in these two cases, the Hamiltonian could be expected to describe properly the boundary bond effect in some real quasi-1D materials at very low temperature, such as the possible bond impurity $S^{\prime}=1 / 2$ in $S$ $=1$ Heisenberg antiferromagnet TMNIN ${ }^{27}$ To show the integrability of the Hamiltonian (7), let us first notice that the impurity term $H_{i m p}$ can be more conveniently treated as the boundary operator, similar to the usual open boundary problem with boundary field. In addition to the Yang-Baxter equation (YBE) as the integrable condition of the bulk, there are some new consistent constraints (often called the reflec- 
tion YBE) for the same model to be integrable under the open boundary conditions, and the QISM is still available. ${ }^{28}$ A new $K$ operator is introduced to describe the boundary effect. In most works, the $K$ operator is a $2 \times 2$ matrix with $c$-number elements that describes the boundary field. ${ }^{28}$ The Sklyanin formalism can be extended to the generic representations of $K$ operator, which is written as a $2 \times 2$ matrix but with elements being operators rather than $c$ numbers. ${ }^{20,21}$ This operator-valued $K$ plays an useful role in constructing the boundary problem where the quantum degrees of freedom of the boundary enter interactions. Of course generally, both $R, K$ matrices could be interpreted as the inhomogeneous vertices in a 2D lattice model. Our model corresponds to a very special one in that the only "inhomogeneity", comes from the boundary row, leaving others uniform. It is built as

$$
K(\lambda)={ }_{S S^{\prime}} R^{A 0}(\lambda-i c)_{S S^{\prime}} R^{A 0}(\lambda+i c)
$$

and ${ }_{S S^{\prime}} R(\lambda)^{A 0}$ is given by

$$
\begin{aligned}
S S^{\prime} R^{A 0}(\lambda)= & -\sum_{l=\left|S-S^{\prime}\right|}^{S+S^{\prime}} \prod_{k=\left|S-S^{\prime}\right|+1}^{l} \frac{\lambda-k}{\lambda+k} \\
& \times \prod_{n \neq l, n=\left|S-S^{\prime}\right|}^{S+S^{\prime}} \frac{y-y_{n}}{y_{l}-y_{n}} .
\end{aligned}
$$

It is straightforward to show that the doubled monodromy matrix

$$
\Theta(\lambda)=T(\lambda) K(\lambda) T^{-1}(-\lambda)
$$

satisfies the reflection YBE and its trace $\theta(\lambda)=\operatorname{tr}_{A} \Theta(\lambda)$ satisfies $[\theta(\lambda), \theta(\mu)]=0, \forall \lambda, \mu$. Similarly, because $H$ $\left.\propto(d / d \lambda) \ln \theta(\lambda)\right|_{\lambda=0}$, the Hamiltonian (7) is indeed integrable. Its spectrum is uniquely determined by the following Bethe ansatz equations (BAE):

$$
\begin{gathered}
\frac{\lambda_{j}+i\left(S^{\prime}+c\right)}{\lambda_{j}-i\left(S^{\prime}+c\right)} \frac{\lambda_{j}+i\left(S^{\prime}-c\right)}{\lambda_{j}-i\left(S^{\prime}-c\right)}\left(\frac{\lambda_{j}+i S}{\lambda_{j}-i S}\right)^{2 N} \\
=\prod_{l \neq j}^{M} \frac{\lambda_{j}-\lambda_{l}+i}{\lambda_{j}-\lambda_{l}-i} \frac{\lambda_{j}+\lambda_{l}+i}{\lambda_{j}+\lambda_{l}-i} .
\end{gathered}
$$

The eigenvalue of the Hamiltonian (7) is

$$
E=-J_{0} \sum_{j=1}^{M} \frac{S}{\lambda_{j}^{2}+S^{2}}
$$

up to a rapidity-independent constant, where $\lambda_{j}$ are the rapidities of the spin waves. The magnetization is given by $S_{z}=N S+S^{\prime}-M$ with $M$ being the number of down spins. For convenience, we put $J_{0}=1$ in the following text.

\section{GROUND STATE, BOUNDARY CORRELATOR, AND BOUNDARY STRINGS}

Due to the reflection symmetry of the model and its BAE, there is a restriction on the rapidities: $\lambda_{j} \neq \pm \lambda_{l}$, for $j \neq l$. Therefore, $\lambda_{j}=0$ is forbidden in this system. Generally, the bulk solutions of Eq. (13) can be described by the following strings in the thermodynamic limit

$$
\lambda_{j, \gamma}^{n}=\lambda_{\gamma}^{n}+\frac{i}{2}(n-2 j+1), \quad j=1,2, \ldots, n,
$$

with $\lambda_{\gamma}^{n}$ being a positive real number. Since $c$ and $-c$ give the same Hamiltonian, we consider $c>0$ ( $c$ real) or $\operatorname{Im} c$ $>0$ ( $c$ imaginary) cases without losing generality. For $c$ $<S^{\prime}$ and or imaginary $c$, Eq. (15) are the only possible solutions of the BAE (13). For each class of states classified by $n$ strings, we introduce the usual density distribution function $\rho_{n}(\lambda)$ and $\rho_{n, h}(\lambda)$, representing occupied states (particles) and missing states (holes), respectively. The BAE of the $n$ strings reads

$$
\begin{aligned}
& \rho_{n, h}(\lambda)+\sum_{l=1}^{\infty} \mathbf{A}_{n l} \rho_{l}(\lambda) \\
& =a_{n, 2 S}(\lambda)+\frac{1}{2 N}\left[\phi_{n}^{i m p}(\lambda)+\phi_{n}^{e d g}(\lambda)\right],
\end{aligned}
$$

where $\quad a_{n}(\lambda)=n / 2 \pi\left(\lambda^{2}+n^{2} / 4\right), \quad a_{n, l}(\lambda)$ $=\sum_{k=1}^{\min (n, l)} a_{n+l+1-2 k}(\lambda) ; \mathbf{A}_{n l}$ is an integral operator with the kernel

$$
A_{n, l}(\lambda)=a_{|n+l|}(\lambda)+2 \sum_{k=1}^{\min (n, l)-1} a_{n+l-2 k}(\lambda)+a_{|n-l|}(\lambda),
$$

$\phi_{n}^{i m p}(\lambda)=a_{n, 2 S^{\prime}}(\lambda-i c)+a_{n, 2 S^{\prime}}(\lambda+i c)$ is the impurity contribution; $\phi_{n}^{e d g}(\lambda)=a_{n}(\lambda)-\delta(\lambda)$ is the surface or edge term, which is independent of the magnetic impurity. Notice that as a direct result of the restriction $\lambda_{j} \neq \lambda_{l}, \delta(\lambda)$ in $\phi_{n}^{e d g}$ excludes the case of $\lambda_{\gamma}^{n}=0$, which corresponds to a vanishing wave function. In the ground state, only $2 S$ strings exist $^{26}$ and Eq. (16) is reduced to

$$
\mathbf{A}_{2 S, 2 S} \rho_{2 S}(\lambda)=a_{2 S, 2 S}(\lambda)+\frac{1}{2 N}\left[\phi_{2 S}^{i m p}(\lambda)+\phi_{2 S}^{e d g}(\lambda)\right] .
$$

By Fourier transforming Eq. (17), we readily obtain

$$
\begin{gathered}
\rho_{2 S}(\lambda)=\rho_{2 S}^{0}(\lambda)+\frac{1}{2 N}\left[\rho_{2 S}^{i m p}(\lambda)+\rho_{2 S}^{e d g}(\lambda)\right], \\
\rho_{2 S}^{0}(\lambda)=\frac{1}{2 \cosh (\pi \lambda)},
\end{gathered}
$$

$$
\rho_{2 S}^{i m p}(\lambda)=\frac{1}{2 \pi} \int \frac{\sinh \left(S^{\prime} \omega\right) \cosh (c \omega)}{\cosh \frac{\omega}{2} \sinh (S \omega)} e^{-i \omega \lambda} d \omega
$$$$
\text { for } S>S^{\prime} \text {, }
$$

$$
\rho_{2 S}^{i m p}(\lambda)=\frac{1}{2 \pi} \int \frac{e^{-\left(S^{\prime}-S\right)|\omega|} \cosh (c \omega)}{\cosh \frac{\omega}{2}} e^{-i \omega \lambda} d \omega
$$

for $S<S^{\prime}$, 


$$
\rho_{2 S}^{e d g}(\lambda)=\frac{1}{2 \pi} \int \frac{\tanh \frac{\omega}{2}\left(1-e^{S|\omega|}\right)}{2 \sinh (S \omega)} e^{-i \lambda \omega} d \omega
$$

The ground-state energy is obtained as

$$
\begin{gathered}
E_{0} / N=f_{\text {bulk }}^{0}+\frac{1}{N}\left(F_{\text {imp }}^{0}+F_{\text {edg }}^{0}\right), \\
f_{\text {bulk }}^{0}=\frac{1}{2}\left[\Psi\left(\frac{1}{2}\right)-\Psi\left(\frac{1}{2}+S\right)\right], \\
F_{\text {imp }}^{0}=\frac{1}{4} \sum_{r= \pm}\left[\Psi\left(\frac{1}{2}+\frac{1}{2}\left|S-S^{\prime}\right|+i r c\right)\right. \\
\left.-\Psi\left(\frac{1}{2}+\frac{1}{2}\left(S+S^{\prime}\right)+i r c\right)\right], \\
F_{e d g}^{0}=\frac{1}{4}\left\{\Psi\left[\frac{1}{2}+\frac{1}{2}\left(S-\frac{1}{2}\right)\right]-\Psi\left[\frac{1}{2}+\frac{1}{2}\left(S+\frac{1}{2}\right)\right]\right\} \\
+\frac{1}{2}\left[\Psi\left(\frac{1}{2} S\right)-\Psi\left(\frac{1}{2} S+1\right)\right],
\end{gathered}
$$

where $\Psi$ is the digamma function. As a by-product, the correlator of $\vec{S}^{\prime}$ and $\vec{S}_{1}$ can be exactly derived for the present model. When $S=1 / 2$ or $S^{\prime}=1 / 2$ we have

$$
\left\langle\vec{S}^{\prime} \cdot \vec{S}_{1}\right\rangle=\frac{\partial}{\partial J} E_{0}
$$

Since the coupling $c$ (and therefore $J$ ) dependent effects are all encoded in $\rho_{2 S}^{i m p}(\lambda)$, we will mainly focus on the impurity energy $F_{i m p}^{0}$ later on. The boundary correlator can be calculated as

$$
\left\langle\vec{S}^{\prime} \cdot \vec{S}_{1}\right\rangle=J^{-2} \frac{\partial}{\partial c^{2}} F_{i m p}^{0}
$$

Now we turn to $c>S^{\prime}$ case. In addition to the $n$-string solutions (15), an imaginary mode $\lambda=i\left(c-S^{\prime}\right)$ appears to be a solution of the BAE (13). In fact, in this case, the socalled $n-k$ boundary string ${ }^{29}$ is a possible solution of the BAE

$$
\lambda_{b s}^{n-k, m}=i\left(c-S^{\prime}\right)+i m, \quad m=k, k+1, \ldots, n,
$$

where $k<S^{\prime}-c$ or $k=0$. Generally, there is no restriction for $n$ in the spin chain with a boundary field. ${ }^{29}$ However, in our model, $\lambda= \pm i\left(S^{\prime}+c\right)$ are not solutions of the BAE, indicating $n<2 S^{\prime}$. In addition, for $2 c=$ integer case, $k$ must be zero due to the restriction $\lambda_{j} \neq \lambda_{l}, j \neq l$. The formation of $n-k$ boundary string in the ground-state configuration will induce a distortion of the original $2 S$ string. The change of the $2 S$-string distribution [denoted by $\rho_{b s}(\lambda)$ ] can be derived from the following equation:

$$
\begin{aligned}
\mathbf{A}_{2 S, 2 S} \rho_{b s}(\lambda)= & -\sum_{l=k}^{n}\left\{a_{2 S, 2 S}\left[\lambda-i\left(c-S^{\prime}+l\right)\right]\right. \\
& \left.+a_{2 S, 2 S}\left[\lambda+i\left(c-S^{\prime}+l\right)\right]\right\} .
\end{aligned}
$$

The energy carried by the boundary string is

$$
\epsilon_{b s}=-\frac{\pi}{2} \int a_{2 S, 2 S}(\lambda) \rho_{b s}(\lambda)-\sum_{l=k}^{n} \frac{S}{S^{2}-\left(c-S^{\prime}+l\right)^{2}} .
$$

By solving Eq. (30) via Fourier transformation and submitting $\rho_{b s}$ into Eq. (31), we find $\epsilon_{b s}=0$. Therefore there is no net contribution from the boundary string to the total energy in the thermodynamic limit, similar to problem of a charged vacuum in the sine-Gordon theory. ${ }^{29}$ However, we remark here that such a kind of boundary string will be stabilized with a finite magnetization, like those of the fermion systems with boundary potential ${ }^{29}$ or Kondo impurity. ${ }^{20,22}$

\section{THERMODYNAMICS}

In this section, we consider the thermodynamics of $c$ $\leqslant S^{\prime}$ (antiferromagnetic) case. The thermodynamic BAE can be derived by following the standard method. ${ }^{8,30,31}$ At finite temperatures, the solutions of BAE are described by Eq. (15). The energy of the system takes the form

$$
\frac{E}{N}=-\pi \sum_{n=1}^{\infty} \int a_{n, 2 S}(\lambda) \rho_{n}(\lambda) d \lambda+\sum_{n=1}^{\infty} n H \int \rho_{n}(\lambda) d \lambda,
$$

where $H$ is the external magnetic field. The entropy of the system reads

$$
\begin{aligned}
S / N= & \sum_{n=1}^{\infty} \int\left\{\left(\rho_{n}+\rho_{n, h}\right) \ln \left(\rho_{n}+\rho_{n, h}\right)-\rho_{n} \ln \rho_{n}\right. \\
& \left.-\rho_{n, h} \ln \rho_{n, h}\right\} d \lambda .
\end{aligned}
$$

By minimizing the free energy $F=E-T S$ we readily obtain the following equation:

$$
\ln \left(1+\eta_{n}\right)=\frac{n H-\pi a_{n, 2 S}}{T}+\sum_{l=1}^{\infty} \mathbf{A}_{n, l} \ln \left(1+\eta_{l}^{-1}\right),
$$

where $\eta_{n}(\lambda) \equiv \rho_{n, h}(\lambda) / \rho_{n}(\lambda)$ and $\eta_{0}(\lambda) \equiv 0$. With the identities

$$
\begin{gathered}
\mathbf{A}_{n, m}-\mathbf{G}\left(\mathbf{A}_{n-1, m}+\mathbf{A}_{n+1, m}\right)=\delta_{n, m}, \quad \mathbf{A}_{1, m}-\mathbf{G} \mathbf{A}_{2, m}=\delta_{1, m}, \\
\mathbf{B}_{n, m}-\mathbf{G}\left(\mathbf{B}_{n-1, m}+\mathbf{B}_{n+1, m}\right)=\delta_{n, m} \mathbf{G} \\
\mathbf{B}_{1, m}-\mathbf{G B}_{2, m}=\delta_{1, m} \mathbf{G}
\end{gathered}
$$

where $\mathbf{B}_{n, m}$ and $\mathbf{G}$ are integral operators with the kernels $a_{n, m}(\lambda)$ and $1 / 2 \cosh (\pi \lambda)$ respectively, Eq. (34) can be reduced to

$$
\begin{aligned}
\ln \eta_{n}= & -\frac{\pi}{2 T \cosh (\pi \lambda)} \delta_{n, 2 S}+\mathbf{G}\left[\ln \left(1+\eta_{n-1}\right)\right. \\
& \left.+\ln \left(1+\eta_{n+1}\right)\right],
\end{aligned}
$$

with the boundary condition

$$
\lim _{n \rightarrow \infty} \frac{\ln \eta_{n}}{n}=\frac{H}{T}
$$


Equation (37) is almost the same equation as that of the $2 S$-channel Kondo problem ${ }^{8}$ with only a different driving term. The free energy reads

$$
\begin{gathered}
F / N=f_{\text {bulk }}+\frac{1}{N} F_{\text {imp }}+\frac{1}{N} F_{e d g}, \\
f_{\text {bulk }}=f_{\text {bulk }}^{0}-T \int[2 \cosh (\pi \lambda)]^{-1} \ln \left[1+\eta_{2 S}(\lambda)\right] d \lambda, \\
F_{e d g}=F_{e d g}^{0}-\frac{1}{2} T \int[2 \cosh (\pi \lambda)]^{-1} \ln \left[1+\eta_{1}(\lambda)\right] d \lambda, \\
F_{\text {imp }}=-S^{\prime} H-\frac{1}{2} T \sum_{n=1}^{\infty} \int \Phi_{n}^{i m p}(\lambda) \ln \left[1+\eta_{n}^{-1}(\lambda)\right] d \lambda .
\end{gathered}
$$

The boundary behaves always as a spin-1/4 (in fact one half of a spin-1/2) and its critical effect in the $X X Z$ spin-1/2 chain has been discussed in a previous work. ${ }^{32}$ In our case, the "boundary spin", shows overscreened critical behavior, as will be discussed in the following section.

When $T \rightarrow 0$, the driving term in Eq. (37) diverges. That means $\eta_{2 S} \rightarrow 0$ and all other $\eta_{n}$ tend to constants $\eta_{n}^{+}$that satisfy the following algebraic equations:

$$
\eta_{n}^{+^{2}}=\left(1+\eta_{n-1}^{+}\right)\left(1+\eta_{n+1}^{+}\right),
$$

with the boundary conditions

$$
\eta_{0}^{+}=\eta_{2 S}^{+}=0, \quad \lim _{n \rightarrow \infty} \frac{\ln \eta_{n}^{+}}{n}=\frac{H}{T} \equiv 2 x_{0} .
$$

Since the equation is decoupled at $n=2 S$, we have different solutions ${ }^{8}$ for $n>2 S$ and $n \leqslant 2 S$

$$
\begin{gathered}
\eta_{n}^{+}=\frac{\sinh ^{2}(n-2 S+1) x_{0}}{\sinh ^{2} x_{0}}-1, \text { for } n \geqslant 2 S, \\
\eta_{n}^{+}=\frac{\sin ^{2} \frac{\pi}{2} \frac{n+1}{S+1}}{\sin ^{2} \frac{\pi}{2(S+1)}}-1, \text { for } n<2 S .
\end{gathered}
$$

The residual entropy of the open boundary is

$$
S_{e d g}=\frac{1}{2} \ln \left[2 \cos \frac{\pi}{2(S+1)}\right] .
$$

To calculate the free energy of the impurity, we rewrite the integral kernel $\Phi_{n}^{i m p}$ in Eq. (42) with real variable as

$$
\begin{aligned}
\Phi_{n}^{i m p}(\lambda)= & {[\alpha]^{-1} a_{n, 2 S^{\prime}-c_{I}}(\lambda)+[\alpha] a_{n, 2 S^{\prime}+c_{I}}(\lambda) } \\
& -a_{\alpha}(\lambda)\left(1-\delta_{\alpha, 0}\right) \sum_{l=1}^{c_{I}} \delta_{n, 2 S^{\prime}-c_{I}+2 l-1},
\end{aligned}
$$

where $c_{I}$ denotes the integer part of $2 c$ and $\alpha=2 c-c_{I}$. For $2 c=c_{I}$, the impurity behaves as two ghost spins $S^{\prime}+c_{I} / 2$ and $S^{\prime}-c_{I} / 2$. The entropy of a ghost spin $\bar{S}$ reads

$$
\begin{aligned}
S_{\text {ghost }}= & \frac{1}{2} \ln [2(\bar{S}-S)+1] \text { for } \quad|\bar{S}|>S, \\
S_{\text {ghost }} & =\frac{1}{2} \ln \frac{\sin \frac{\pi}{2} \frac{2 \bar{S}+1}{\sin \frac{\pi}{2(S+1)}} \text { for }|\bar{S}| \leqslant S .}{} \text {. }
\end{aligned}
$$

The summation of the two ghost spins' entropy gives that of the whole impurity. When $c_{I} \neq 2 c$, the difference between the residual entropies for $c_{I} \neq 2 c$ and $c_{I}=2 c$, i.e., $\Delta S_{i m p}$ reads

$$
\Delta S_{i m p}=\frac{1}{2} \sum_{l=1}^{c_{I}}\left[\ln \left(1+f_{2 S^{\prime}-c_{I}+2 l-1}\right)-\ln f_{2 S^{\prime}-c_{I}+2 l-1}\right],
$$

with $f_{n}=\lim _{x_{0} \rightarrow 0} \eta_{n}^{+}$. This result shows that the spin configuration of the ground state is very complicated and strongly depends on the impurity-bulk coupling. In fact, the residual entropy has jumps at $c=c_{I} / 2$. That means quantum phase transition occurs for $c$ across a half-integer or an integer.

To obtain the leading order of some thermodynamic quantities such as the specific heat and the susceptibility, we need the low-temperature $\left(T \ll T_{k}\right)$ expansion. This can be achieved by following the standard method developed for the multichannel Kondo problem. ${ }^{8}$ For $T \rightarrow 0$, only the excitations near the Fermi surface $(\lambda \rightarrow \pm \infty)$ are important. The driving term in Eq. (37) can be approximately replaced by $-(\pi / T) \exp (-\pi|\lambda|)$. We introduce the new variables $\zeta_{ \pm}=$ $\pm \pi \lambda+\ln (\pi / T)$, then $\eta_{n}$ takes the following asymptotic forms: ${ }^{8}$

$$
\begin{aligned}
& \eta_{n}\left(\zeta_{ \pm}\right) \sim \eta_{n}^{+}+\left(\alpha_{n}+\beta_{n} x_{0}^{2}\right) e^{-\zeta_{ \pm}} \text {for } n \geqslant 2 S, \\
& \eta_{n}\left(\zeta_{ \pm}\right) \sim \eta_{n}^{+}+\left(\alpha_{n}+\beta_{n} x_{0}^{2}\right) e^{-\tau \zeta_{ \pm}} \text {for } n<2 S . \\
& \eta_{1}\left(\zeta_{ \pm}\right) \sim \eta_{1}^{+}+\left(\alpha_{1}+\beta_{1} x_{0}^{2}\right) \zeta_{ \pm} e^{-\tau \zeta_{ \pm}} \text {for } S=1 .
\end{aligned}
$$

Here $\alpha_{n}$ and $\beta_{n}$ are constants, $\tau=2 /(S+1)$ and \pm denotes the two Fermi points. For imaginary $c=i b$, the free energy of the impurity reads

$$
\begin{aligned}
F_{i m p} \sim F_{i m p}^{0}-\frac{1}{2} T \int\left[\frac{1}{2 \cosh \left(\zeta+\pi b-\ln \frac{\pi}{T}\right)}\right. \\
\left.+\frac{1}{2 \cosh \left(\zeta-\pi b-\ln \frac{\pi}{T}\right)}\right] \ln \left(1+\eta_{2 S^{\prime}}\right) d \zeta .
\end{aligned}
$$

Notice that we have replaced $\zeta_{ \pm}$by $\zeta$ in the integral. In this case, the bond deformation does not change the effective strength of the impurity but the energy scale $T_{k}$ (Kondo temperature $)^{21}$

$$
T_{k} \sim \pi \cosh ^{-1}(\pi b)
$$


The system behaves as a $2 S$-channel Kondo system with an impurity $\vec{S}^{\prime}$. For $c$ real and $2 c=c_{I}$, the free energy of the impurity can be rewritten as

$$
F_{i m p} \sim F_{i m p}^{0}-\frac{1}{2} T \sum_{ \pm} \int \frac{\ln \left(1+\eta_{2 S^{\prime} \pm c_{I}}\right)}{2 \cosh \left(\zeta-\ln \frac{\pi}{T}\right)} d \zeta .
$$

For $c_{I} \neq 2 c$,

$$
\begin{aligned}
F_{i m p} \sim F_{i m p}^{0}-T \sum_{ \pm} \int G_{ \pm}\left[\frac{1}{\pi}\left(\zeta-\ln \frac{\pi}{T}\right)\right] \ln \left(1+\eta_{2 S^{\prime} \pm c_{I}}\right) d \zeta \\
+\frac{1}{2} T \sum_{l=1}^{c_{I}} \int a_{\alpha}(\lambda) \ln \left(1+\eta_{2 S^{\prime}-c_{I}+2 l-1}^{-1}\right) d \zeta
\end{aligned}
$$

where

$$
G_{ \pm}(\lambda)=\int \frac{e^{\mp\left[c-(1 / 2) c_{I}\right]|\omega|}}{4 \pi \cosh \frac{\omega}{2}} e^{-i \lambda \omega} d \omega .
$$

Notice that $G_{ \pm}(\lambda)$ are convergent in the real axis since $c$ $-c_{I} / 2<1 / 2$. The specific heat and the susceptibility can be easily derived from the free energy by substituting Eqs. (52)-(54) into Eqs. (57) or (58). With different values of $c$, different quantum critical behavior may appear. (i) For $S$ $\pm c_{I} / 2 \geqslant S$, both of the ghost spins are underscreened and the leading terms in the specific heat and the susceptibility are the Schottky term and the Curie term, respectively. (ii) For $S^{\prime}-c_{I} / 2<S<S^{\prime}+c_{I} / 2$, no matter how large $S^{\prime}$ is, the larger ghost spin is underscreened and the smaller ghost spin is overscreened. The leading order of the impurity specific heat is governed by the smaller ghost spin that shows an anomalous power law

$$
C_{i m p} \sim T^{\tau}, \quad \tau=\frac{2}{S+1},
$$

while that of the susceptibility of the impurity is governed by the larger ghost spin with the usual Curie law

$$
\chi_{i m p} \sim T^{-1}+O\left(T^{\tau-1}\right),
$$

indicating a novel critical behavior. (iii) For $S^{\prime} \pm c_{I} / 2<S$, the system behaves as a conventional overscreened Kondo system. Especially for $S=1, S^{\prime}=1 / 2$, and $c_{I}=0$, the model is related to Eq. (1) and the low-temperature behavior of the impurity shows as

$$
C_{i m p} \sim-T \ln T, \quad \chi_{i m p} \sim-\ln T,
$$

which coincides with the numerical results obtained in Ref. 25 . The above results show that the bond deformation leads to two kinds of effect: The half integer (or integer) part of $c$ $\left(c_{I} / 2\right)$ splits the impurity spin into two ghost spins $\left(S^{\prime}\right.$ $\left.\pm c_{I} / 2\right)$, and the residue $\left(c-c_{I} / 2\right)$ renormalizes the effective energy scale $T_{k}$ (Kondo temperature) as well as the amplitudes of the physical quantities (but without changing the critical behavior). Thus the critical behavior is only governed by the integer part of $2 c$.

\section{CONCLUDING REMARKS}

In conclusion, we propose an integrable model of a boundary impurity spin $\vec{S}^{\prime}$ coupled with an open Takhatajian-Babujian spin- $S$ chain. The relation between the present model and the problem of the bulk impurity in a spin chain is discussed. In our model, when $S$ or $S^{\prime}$ is one half, the interaction term takes a very simple form. The coupling constant $J$ can take arbitrary value without destroying the integrability of the Hamiltonian, this allows one to exhibit the exact features of the system. While in the periodic models, there is a constraint on $J$. Though a similar ${ }_{S^{\prime}} R^{A 0}(\lambda$ $-c$ ) can be introduced in the periodic models, ${ }^{6,5}$ the parameter $c$ must be real (imaginary in our case) which describes a weak-linked impurity with the bulk. The interaction only affects the energy scale (Kondo temperature) but does not change the fixed point of the system. With an imaginary $c$, the model Hamiltonians constructed for bulk impurities are non-Hermitian and their spectra generally lie in the complex plane ${ }^{33}$ rather than in the real axis. In our model, both real $c$ and imaginary $c$ define Hermitian Hamiltonians due to the reflection symmetry, and the coupling constant $J$ meets all physical situations. Some new quantum critical phenomena driven by the impurity-bulk coupling have been found (which have not been explicitly found yet in the periodic models): (i) The strong coupling $J$ may split the impurity spin into effective "ghost spins" $S^{\prime}-c_{I} / 2$ and $S^{\prime}+c_{I} / 2$. The coupling not only changes the energy scales (Kondo temperature) as in the conventional Kondo problem but also renormalizes the effective strength of the impurity spin. Such a phenomenon reveals a pure correlation effect. We note that the spin splitting or the ghost spins have also been pointed out in our earlier publications in some different situations. ${ }^{20,22}$ (ii) Depending on the strength of the coupling, the system may show a variety of critical behaviors, which are different from those of the conventional Kondo problem. A typical example is that when $S^{\prime}-c_{I} / 2<S<S^{\prime}$ $+c_{I} / 2$, the leading term in the susceptibility is Curie type (contributed by the larger ghost spin), while that of the specific heat is an overscreened $2 S$-channel Kondo type (contributed by the smaller ghost spin). Such a fascinating nonFermi liquid behavior has never been found in the conventional impurity problem (notice that they are induced by the same impurity). (iii) The open boundary, which can be produced by either an impurity (magnetic or nonmagnetic) or bond deformation, shows overscreened multichannel behavior as long as the bulk spin $S>1 / 2$. Such kind of effect is caused by the self-avoiding scattering of a spin wave with its reflection counterpart, and is expected to be a common feature of the multichannel systems in one dimension. Our results strongly suggest that some new intermediate fixed points may exist for the Kondo problem in a strongly correlated system.

\section{ACKNOWLEDGMENTS}

Two of the authors (J.D., Y.W.) are grateful to Professor N. Andrei and Professor Yu Lu for helpful discussions. Y.W. also acknowledges the financial support of AvH-Stiftung, NSFC, FCAS, and NOYSFC, National Natural Science Foundation of China. 
${ }^{1}$ A. O. Gogolin, Ann. Phys. (Paris) 19, 411 (1994).

${ }^{2}$ K. Moon, C. L. Kane, S. M. Givin, and M. P. A. Fisher, Phys. Rev. Lett. 71, 4381 (1993).

${ }^{3}$ S. Iijima, Nature (London) 354, 56 (1991); S. Tans, M. H. Devoret, H. Dai, A. Thess, R. E. Swalley, L. J. Geerligs, and C. Dekker, ibid. 386, 474 (1997).

${ }^{4}$ J. Voit, Rep. Prog. Phys. 57, 977 (1994).

${ }^{5}$ N. Andrei and H. Johannesson, Phys. Lett. 100A, 108 (1981); G. Bedürftig, F. H. Eßler, and H. Frahm, Phys. Rev. Lett. 77, 5098 (1996); P. Schlottmann, Int. J. Mod. Phys. B 11, 355 (1997); P. Schlottmann and A. A. Zvyagin, Phys. Rev. B 55, 5027 (1997); Nucl. Phys. B: Field Theory Stat. Syst. 501[FS], 728 (1997); Phys. Rev. B 56, 13989 (1997); A. A. Zvyagin and P. Schlottmann, J. Phys.: Condens. Matter 9, 3543 (1997); Phys. Rev. B 56, 300 (1997).

${ }^{6}$ K.-J.-B. Lee and P. Schlottmann, Phys. Rev. B 37, 379 (1987); P. Schlottmann, J. Phys.: Condens. Matter 3, 6619 (1991).

${ }^{7}$ P. Schlottmann, Phys. Rev. Lett. 54, 2131 (1985); M. Takahashi and M. Yamada, J. Phys. Soc. Jpn. 54, 2808 (1985).

${ }^{8}$ N. Andrei and C. Destri, Phys. Rev. Lett. 52, 364 (1984); for a review, see N. Andrei, in Low-Dimensional Quantum Field Theories for Condensed Matter Physics, edited by S. Lundqvist, G. Morandi, and Yu Lu (World Scientific, Singapore, 1995).

${ }^{9}$ C. L. Kane and M. P. A. Fisher, Phys. Rev. Lett. 68, 1220 (1992); Phys. Rev. B 46, 15233 (1992).

${ }^{10}$ D.-H. Lee and J. Toner, Phys. Rev. Lett. 69, 3378 (1992).

${ }^{11}$ A. Furusaki and N. Nagaosa, Phys. Rev. Lett. 72, 892 (1994).

${ }^{12}$ P. Fröjdh and H. Johannesson, Phys. Rev. Lett. 75, 300 (1995).

${ }^{13}$ J. L. Cardy, Nucl. Phys. B 240, 514 (1984); 324, 581 (1984).

${ }^{14}$ A. W. W. Ludwig and I. Affleck, Nucl. Phys. B 428, 545 (1994).

${ }^{15}$ S. Eggert and I. Affleck, Phys. Rev. B 46, 10866 (1992).

${ }^{16}$ H. J. de Vega and F. Woynarovich, J. Phys. A 25, 4499 (1992); H. J. de Vega, L. Mezincesan, and R. I. Nepomechie, Phys. Rev. B 49, 13223 (1994).

${ }^{17}$ S. Qin, M. Fabrizio, and Lu Yu, Phys. Rev. B 54, 9854 (1996); S.
Qin, M. Fabrizio, Lu Yu, M. Oshikawa, and I. Affleck, ibid. 56, 9766 (1997).

${ }^{18}$ E. S. Sorensen, S. Eggert, and I. Affleck, J. Phys. A 26, 7657 (1993).

${ }^{19}$ Y. Wang, J. Voit, and F.-C. Pu, Phys. Rev. B 54, 8491 (1996).

${ }^{20}$ Y. Wang, J. H. Dai, Z. N. Hu, and F. C. Pu, Phys. Rev. Lett. 79, 1901 (1997); see also A. Zvyagin, ibid. 79, 5703 (1997).

${ }^{21}$ Y. Wang, Phys. Rev. B 56, 14045 (1997); H. Frahm and A. Zvyagin, J. Phys.: Condens. Matter 9, 9939 (1997).

${ }^{22}$ Y. Wang and U. Eckern, Phys. Rev. B 59, 6400 (1999).

${ }^{23}$ Y. Wang and F.-C Pu, Chin. Phys. Lett. 13, 703 (1996); M. Fabrizio and A. O. Gogolin, Phys. Rev. B 51, 17827 (1995); V. J. Emery and S. Kivelson, ibid. 46, 10812 (1992); Phys. Rev. Lett. 71, 3701 (1993).

${ }^{24}$ N. Andrei and A. Jerez, Phys. Rev. Lett. 74, 4507 (1995); P. Schlottmann and P. D. Scramento, Adv. Phys. 42, 641 (1993).

${ }^{25}$ S. Eggert and S. Rommer, Phys. Rev. Lett. 81, 1690 (1998); Physica B 261, 200 (1999).

${ }^{26}$ L. Takhatajian, Phys. Lett. 87A, 479 (1982); H. M. Babujian, Nucl. Phys. B 215, 317 (1983); H. M. Babujian and T. M. Tsvelick, ibid. 265, 24 (1986); P. P. Kulish, N. Yu Reshetikhin, and E. K. Sklyanin, Lett. Math. Phys. 5, 393 (1981).

${ }^{27}$ T. Kawae, M. Ito, M. Mito, M. Hitaka, and K. Takeda, J. Phys. Soc. Jpn. 66, 1892 (1997).

${ }^{28}$ E. K. Sklyanin, J. Phys. A 21, 2375 (1988).

${ }^{29}$ A. Kapustin and S. Skorik, J. Phys. A 29, 1629 (1996); S. Skorik and H. Saleur, ibid. 28, 6605 (1995); G. Bedürftig and H. Frahm, ibid. 30, 4139 (1997).

${ }^{30}$ C. N. Yang and C. P. Yang, J. Math. Phys. 10, 1115 (1969).

${ }^{31}$ M. Takahashi, Prog. Theor. Phys. 46, 401 (1971); 46, 1388 (1971); M. Gaudin, Phys. Rev. Lett. 26, 1301 (1971).

${ }^{32}$ P. de Sa and A. M. Tsvelik, Phys. Rev. B 52, 3067 (1995).

${ }^{33}$ I. Y. Goldsheid and B. A. Khoruzhenko, Phys. Rev. Lett. 80, 2897 (1998); N. M. Shnerb and D. R. Nelson, ibid. 80, 5172 (1998). 